\title{
IMPROVEMENTS OF BOUNDS FOR THE $q$-GAMMA AND THE $q$-POLYGAMMA FUNCTIONS
}

\author{
Ahmed Salem And FARis Alzahrani
}

Abstract. In this paper, the complete monotonicity property of functions involving the $q$-gamma function is proven and used to establish sharp inequalities for the $q$-gamma and the $q$-polygamma functions for all $q>0$. These bounds for the $q$-gamma and the $q$-polygamma functions refine those given by Salem [17].

Mathematics subject classification (2010): 33D05, 26D07, 26A48.

Keywords and phrases: Inequalities, $q$-Gamma function, $q$-polygamma functions, completely monotonic function.

\section{REFERENCES}

[1] D. S. MoAK, The q-gamma function for $q>1$, Aequationes Math., vol. 20 (1980) 278-285.

[2] C. Krattenthaler, H. M. SRivastaVA, Summations for basic hypergeometric series involving a q-analogue of the digamma function, Computers Math. Applic., vol. 32 (2) (1996) 73-91.

[3] A. SAlEM, Some Properties and Expansions Associated with the q-Digamma Function, Quaestiones mathematicae, vol. 36 (1) (2013) 67-77.

[4] Z. Cheng, Bose-Einstein condensation of ideal photons in a one-dimensional barrel cavity, Physical Review A, vol. 93 (2) 023829 (2016).

[5] Z. CHENG, Exact spatial density of ideal Bose atoms in a one-dimensional harmonic trap, Journal of Statistical Mechanics: Theory and Experiment, vol. 2016 (5) 053102 (2016).

[6] Z. CHENG, Exact thermodynamic theory of an ideal boson gas in a one-dimensional harmonic trap, Journal of Statistical Mechanics: Theory and Experiment, vol. 2015 (11) P11003 (2015).

[7] N. BATIR, Monotonicity properties of q-digamma and q-trigamma functions, Journal of Approximation Theory, vol. 192 (7) (2015) 336-346.

[8] N. BATIR, $q$-Extensions of some estimates associated with the digamma function, Journal of Approximation Theory, vol. 174 (2013) 54-64.

[9] A. SALEM, Completely monotonic functions related to the gamma and the q-gamma functions, Revista de la Real Academia de Ciencias Exactas, Fisicas y Naturales - Serie A: Matematicas, vol. 111 (1) (2017) 271-280.

[10] A. SALEM, A certain class of approximations for the q-digamma function, Rocky Mountain Journal of Mathematics, vol. 45 (5) (2016) 1665-1677.

[11] A. SALEM, Some classes of completely monotonic functions related to q-gamma and q-digamma functions, Mathematical Inequalities \& Applications, vol. 19 (3) (2016) 853-862.

[12] A. SALEM, Monotonic functions related to the q-gamma function, Monatshefte fur Mathematik, vol. 179 (2) (2016) 281-292.

[13] A. SALEM, Completely monotonic functions related to the $q$-gamma and the $q$-trigamma functions, Analysis and Applications, vol. 13 (2) (2015) 125-134.

[14] A. SAlEm, On the q-beta function inequalities, Mathematical Inequalities \& Applications, vol. 18 (2) (2015) 639-648.

[15] A. SALEM, Two classes of bounds for the q-gamma and the q-digamma functions in terms of the $q$-zeta functions, Banach Journal of Mathematical Analysis, vol. 8 (1) (2014) 109-117.

[16] A. SALEM, Complete monotonicity properties of functions involving q-gamma and q-digamma functions, Mathematical Inequalities \& Applications, vol. 17 (3) (2014) 801-811. 
[17] A. SALEM, An infinite class of completely monotonic functions involving the q-gamma function, Journal of Mathematical Analysis and Applications, vol. 406 (2) (2013) 392-399.

[18] A. SALEM, A completely monotonic function involving q-gamma and q-digamma functions, Journal of Approximation Theory, vol. 164 (7) (2012) 971-980.

[19] P. GAO, Some Monotonicity Properties of Gamma and q-Gamma Functions, ISRN Mathematical Analysis, vol. 2011 (2011) 1-15.

[20] H. AlZer, A. Z. GRInshPan, Inequalities for the gamma and q-gamma functions, Journal of Approximation Theory, vol. 144 (2007) 67-82.

[21] A. Z. GRINShPAN AND M. E. H. Ismail, Completely monotonic functions involving the gamma and q-gamma functions, Proc. Amer. Math. Soc., vol. 134 (2006) 1153-1160.

[22] N. Elezovic, C. Giordano and J. Pecaric, Convexity and q-gamma function, Rendiconti del Circolo Matematico di Palermo, vol. serie II, tomo XLVIII (1999) 285-298.

[23] M. E. H. Ismail AND M. E. Muldoon, Inequalities and monotonicity properties for gamma and q-gamma functions, in: R. V. M. Zahar (Ed.), Approximation and Computation, International Series of Numerical Mathematics, vol. 119 Birkhauser, Boston, MA (1994) 309-323.

[24] M. E. H. Ismail, L. LorCh AND M. E. Muldoon, Completely monotonic functions associated with the gamma function and its q-analogues, Journal of Mathematical Analysis and Applications, vol. 116 (1986) 1-9.

[25] D. S. MOAK, The q-analogue of Stirling's formula, Rocky Mountain J. Math., vol. 14 (1984) $403-$ 413.

[26] F. QI AND B.-N. GUO, Complete monotonicities of functions involving the gamma and digamma functions, RGMIA Res. Rep. Coll., vol. 7 (1) (2004) 63-72.

[27] C. BERG, Integral representation of some functions related to the gamma function, Mediterr. J. Math., vol. 1 (4) (2004) 433-439.

[28] B.-N. GUO AND F. QI, A property of logarithmically absolutely monotonic functions and the logarithmically complete monotonicity of a power-exponential function, Politehn. Univ. Bucharest Sci. Bull. Ser. A Appl. Math. Phys., vol. 72 (2) (2010) 21-30.

[29] F. QI, C.-P. CHEN, A complete monotonicity property of the gamma function, Journal of Mathematical Analysis and Applications, vol. 296 (2004) 603-607.

[30] F. QI AND Q.-M. LUO, Bounds for the ratio of two gamma functions-From Wendel's and related inequalities to logarithmically completely monotonic functions, Banach J. Math. Anal., vol. 6 (2) (2012) $132-158$.

[31] M. Abramowitz, C. A. Stegun, Handbook of Mathematical functions with formulas, Graphs, Mathematical tables 7th printing, Applied Mathematics Series, vol. 55, Nathional Bureau of standards, Washington, DC, 1964. 